\title{
Fast Logical Location of Faults in Large Analog Electronic Circuits
}

$\begin{array}{lll}\text { Non-member } & \text { Cai Jinding } & \text { (Fuzhou University, China) } \\ \text { Member } & \text { Chen Shuo } & \text { (Fuzhou University, China) } \\ \text { Member } & \text { Mineo Tsuji } & \text { (Nagasaki University, Japan) } \\ \text { Non-member } & \text { Ma Xikui } & \text { (Xi' an Jiaotong University, China) } \\ \text { Non-member } & \text { Huang Dongquan } & \text { (Fuzhou University, China) }\end{array}$

This paper presents a method for fast off-line logical location of faults in analog electronic circuits at the sub-network level and verifies its practical diagnosability. The proposed approach breaks through the previous limitation that all torn terminals (incident nodes) must be accessible and that the mutual-testing method must be utilized to locate the faulty sub-networks. As far as the diagnosability is concerned, its application is more extensive than the unified decomposition approach. Therefore it better satisfies the engineering needs

Keywords: analog circuit, sub-network, off-line fault diagnosis, intersected-torn rule, logical location

\section{Introduction}

In general, analog electronic circuits are diagnosed at offline state. Topological constructions of practical analog electronic circuits should almost never be satisfied with the conditions of diagnosability for the number of faulty elements $k>2$ when applying node-faulty equations to locate the faulty element ${ }^{[1]}$. Other diagnosis methods have similar conclusions $[2,3]$. Therefore, fault diagnosis theory at the sub-network level has received special attention recently ${ }^{[4]}$. However, up to now the above methods for diagnosing at the sub-network level almost limit to all torn nodes (incident nodes) to be accessible and apply the mutual-testing method to locate the faulty sub-networks. Apparently, these restraining conditions are comparatively rigorous and limit its applications ${ }^{[1]}$

This paper presents a new off-line torn search approach at sub-network level that breaks through the above-restrained conditions, and applies the logical diagnostic function or logical diagnostic matrix to locate the faulty sub-network. Here we also verify in detail its diagnosability as compared with the unified decomposition approach.

\section{Intersected-Torn Search Approach}

\section{$\langle 2 \cdot 1\rangle$ Intersected-torn rules According to the} physical constructions and technological requirements of electronic circuits, we can divide a network $N$ into many subnetworks $S_{i}(i=1,2, \ldots)$, as shown in Fig. 1 (a). If an incident node between two sub-networks can be described as a line, the incidence relations between sub-networks can be described as a torn diagnostic graph $\mathrm{TP}$, as shown in Fig. 1 (b).

For the first tearing $T_{1}$ in Fig.1 (b), TP can be performed along the boundary between $\left\{S_{1}, S_{3}\right\}$ and $\left\{S_{2}, S_{4}, S_{5}\right\}$. Then, we can obtain the sub-networks sets $N_{1}^{1}$ and $\hat{N}_{1}^{1}$, as shown in Fig. 2 (a) and (b). The second tearing $T_{2}$ can be performed

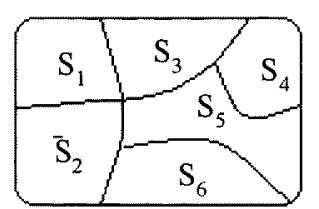

(a) Network $N$

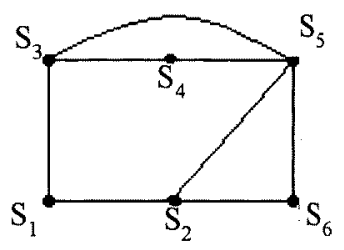

(b) TP graph
Fig. 1. Network connection

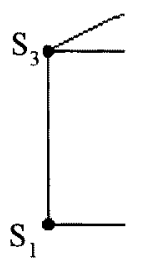

(a) $N_{1}^{1}$

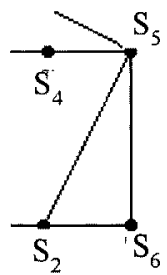

(b) $\hat{N}_{1}^{1}$
Fig. 2. First tearing $T_{1}$

along the boundary between $\left\{S_{1}, s_{2}\right\}$ and $\left\{S_{3}, s_{5}, s_{6}\right\}$. We can obtain the sub-networks sets $N_{1}^{2}$ and $\hat{N}_{1}^{2}$, as shown in Fig. 3 (a) and (b). By repeating the tearing, we can obtain the sub-networks sets $N_{1}^{1}, \hat{N}_{1}^{1}, N_{1}^{2}, \hat{N}_{1}^{2}$, and so on. By selecting the accessible nodes correctly for these subnetworks ${ }^{[4,5]}$, we can judge whether or not each sub-network has a fault.

Let $N$ be the linear network in Fig.1 (a), tearing $N$ into two sub-networks: $N_{1}^{1}$ and $\hat{N}_{1}^{1}$. In $N_{1}^{1}$ (as shown in Fig.4), 


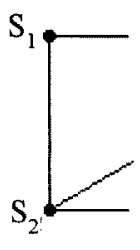

(a) $N_{1}^{2}$

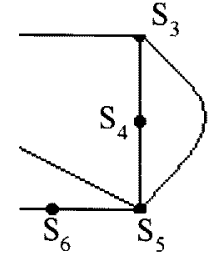

(b) $\hat{N}_{1}^{2}$
Fig. 3. Second tearing $T_{2}$

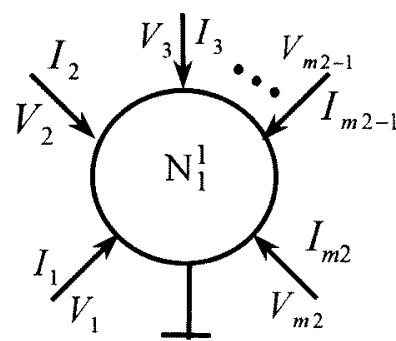

Fig. 4. Sub-network

the torn nodes consist of the inaccessible subset $T T$ ( $t t \in T T$ ) and the accessible subset $M T$ ( $m t \in M T$ ), and the un-torn accessible nodes by $G M(g m \in G M)$ ). Then the following equations can be obtained.

$$
\begin{aligned}
& m=g m+m t \\
& m_{2}=t t+m t
\end{aligned}
$$

where $m$ and $m_{2}$ are respectively the number of accessible nodes and the number of torn nodes in $N_{1}^{\mathrm{l}}$. If $N_{1}^{1}$ is connected and not mutual-coupled with $\hat{N}_{1}^{1}$ in Fig.4, the node-voltage equation of $N_{1}^{1}$ is expressed as

$$
\left[Y_{1 n}\right]\left[V_{1 n}\right]=\left[I_{m}\right]+\left[\begin{array}{c}
I_{t s} \\
0
\end{array}\right]
$$

where, $\left[V_{1 n}\right]=\left[\begin{array}{llll}V_{T T} & V_{M T} & V_{G M} & V_{I I}\end{array}\right]^{T}$,

$$
\begin{aligned}
& {\left[I_{m}\right]=\left[\begin{array}{llll}
0 & I_{M T} & I_{G M} & 0
\end{array}\right]^{T},} \\
& {\left[I_{t s}\right]=\left[\begin{array}{ll}
I_{T T} & I_{S M}
\end{array}\right]^{T}}
\end{aligned}
$$

Here $\left[Y_{1 n}\right]$ is determined by the nominal values of fault-free elements in $N_{1}^{1}$ and $\left[I_{S M}\right]$ is the vector of unknown currents at the torn accessible nodes. Eliminating the vector of internal node-voltage $\left[V_{I I}\right]$ from (3) and rearranging some elements of $\left[V_{1 n}\right]$, we obtain the following equation.

$$
\left[\begin{array}{ll}
Y_{1} & Y_{2} \\
Y_{3} & Y_{4}
\end{array}\right]\left[\begin{array}{l}
V_{m} \\
V_{i}
\end{array}\right]=\left[I_{m}\right]+\left[\begin{array}{c}
I_{t s} \\
0
\end{array}\right]
$$

where $\left[V_{m}\right]=\left[\begin{array}{ll}V_{M T} & V_{G M}\end{array}\right]^{T}:$ the known vector,

$$
\left[V_{i}\right]=\left[\begin{array}{ll}
V_{T T} & V_{I I}
\end{array}\right]^{T}: \text { the unknown vector. }
$$

Taking the measured voltage values of accessible node $\left[V_{m}\right]$ into (4) when $m=m_{2}$, the unknown vector $\left[I_{t s}\right]$ can be calculated by (4). If $N_{1}^{1}$ is fault-free, $\left[I_{t s}\right]$ should satisfy the Kirchhoff current law, i.e.

$$
\sum_{j=1}^{m_{2}} I_{s_{j}}=0
$$

If $N_{1}^{i}$ of the $i$ th-time tearing is satisfied with (5), all subnetworks of $N_{1}^{i}$ are fault-free, and its logical value is 0 , i.e. $\operatorname{TS}\left(N_{1}^{i}\right)=0$. Otherwise, one or more sub-networks of $N_{1}^{i}$ may be faulty and its logical value is 1 , i.e. $T S\left(N_{1}^{i}\right)=1$. Therefore, each sub-network can be associated with a logical variable that takes the value 0 if the sub-network is fault-free and 1 if it is possibly faulty

Although a given electronic circuit may contain several hundred components, it is reasonable to assume that at most two or three components have failed simultaneously. So our research is based on the assumption that the number of faulty sub-networks $f$ should never be greater than 3 . Namely, we shall derive the intersected-torn searching rules under $f \leq 3$ [6].

DEFINITION If a network $N$ may be divided into $m$ subnetworks, any two or any three sub-networks can consist of a pseudo-two sub-networks $S_{i j}$ or a pseudo-three sub-networks $S_{i j k}$ respectively. The sum of the pseudo sub-networks is obviously $C_{m}^{2}+C_{m}^{3}$

Now illustrated with Fig.2, we investigate how the information of non-faulty sub-networks or non-faulty pseudo sub-networks can be obtained from the logical variables in each tearing. Let the logical variables in the first tearing $T_{1}$ for Fig. 2 be $T S\left(N_{1}^{1}\right)=0$ and $T S\left(\hat{N}_{1}^{1}\right)=1$. If $f=1$, the nonfaulty sub-networks should be $s_{1}$ and $s_{3}$ and the possibly faulty sub-networks should be $S_{2}, S_{4}, S_{5}$ and $S_{6}$, respectively. If $f=2$, the non-faulty and the possibly faulty pseudo-two sub-networks should be $s_{12}, s_{13}, s_{14}, s_{15}, s_{16}, s_{23}, s_{34}, s_{35}, s_{36}$ and $s_{24}, s_{25}, s_{26}, S_{45}, s_{46}, s_{56}$. If $f=3$, we can also obtain the information about the pseudo-three sub-networks (to be omitted). The above method for obtaining information about sub-networks and pseudo sub-networks can also be applied to the second tearing $T_{2}$, the third tearing $T_{3}$ and so on. According to this logical reasoning process, we can present four rules for tearing a network $N$ under $f \leq 3$ as follows:

RULE 1 Any two sub-networks $S_{i}$ and $s_{j}$ of network $N$ have been torn at least once, during cross tearing of $k$ times during which the sub-network set $N_{1}^{i}$ or $\hat{N}_{1}^{i}(i=1,2, \ldots k)$ includes one and only one of the two sub-networks $S_{j}$ or $S_{j}$

RULE 2 Any two pseudo-two sub-networks $S_{i j}$ and $S_{p t}$ of $N$ 
have been torn at least once, during cross tearing of $k$ times during which $N_{1}^{i}$ or $\hat{N}_{1}^{i}(i=1,2, \ldots k)$ includes one and only one of the two pseudo-two $s_{i j}$ or $s_{p i}$

RULE 3 Any two pseudo-three sub-networks $s_{i j k}$ and $s_{p q r}$ of $N$ have at least been torn once, during cross tearing of $k$ times during which $N_{1}^{i}$ or $\hat{N}_{1}^{i}(i=1,2, \ldots k)$ includes only one of the two pseudo-three $s_{i, k}$ or $s_{p q r}$ but not the other one.

RULE 4 During cross tearing of $k$ times, any three of the sub-networks $s_{1}, s_{j}, s_{k}$ of $N$ have been torn at least three times which make them belong to $N_{1}^{i}$ or $\hat{N}_{1}^{i}$ separately.

Obviously, rules 1 and 2 are in need of $f \leq 2$ and rules 14 are in need of $f \leq 3$.

$\langle 2 \cdot 2\rangle$ Logical Location of faulty sub-networks

Network $N$ has been torn k times satisfying the above rules, the fault of the sub-networks should be located by $L D_{f}(f$ $=1,2,3)$ referred to as the logical diagnostic matrix. The matrix $L D_{f}$ is formed as below. The rows of $L D_{f}$ correspond to the number of tearings $T_{i}(i=1,2, \ldots, k)$, and the columns of $L D_{f}$ correspond to the sub-networks under $f=1$ or to pseudotwo sub-networks under $f=2$ or to pseudo-three sub-networks under $f=3$. The elements of $L D_{f}$ are 0 or 1 . If the sub-networks or the pseudo sub-networks correspond to the $j$ column of the matrix in $T_{i}$ tearing, then it is judged to be fault-free, the element $(i, j)$ of the matrix is 0 , and 1 otherwise.

Now the matrix $L D_{f}$ formation is illustrated with the first tearing $T_{l}$ of Fig. 2. If $N_{1}^{1}$ is judged non-faulty, then all subnetworks in $N_{1}^{1}$ are non-faulty. Otherwise, $\hat{N}_{1}^{1}$ is faulty. Some sub-networks in $\hat{N}_{1}^{1}$ may be faulty. The matrix $L D_{f}$ formation is discussed as follows:

1) If there is a sub-network fault in networks $N(f=1)$, then non-faulty sub-networks are $S_{1}, S_{3}$ and possible faulty subnetworks are $s_{2}, s_{4}, S_{5}, S_{6}$. The elements of the matrix $L D_{1}$ are deduced as follows:

$$
L D_{1}=\left[\begin{array}{cccccc}
s_{1} & s_{2} & s_{3} & s_{4} & s_{5} & s_{6} \\
0 & 1 & 0 & 1 & 1 & 1 \\
& & \vdots & & &
\end{array}\right]
$$

2) If there are two faulty sub-networks in networks $N(f=2)$, then the non-faulty pseudo-two sub-networks are $s_{12}, s_{13}, S_{14}$, $s_{15}, s_{16}, S_{23}, S_{34}, s_{35}, S_{36}$ and possible faulty pseudo-two subnetworks are $s_{24}, S_{25}, S_{26}, S_{45}, S_{46}, S_{56}$. The matrix $L D_{2}$ is formed as below.

$$
\begin{gathered}
L D_{2}=\left[\begin{array}{ccccccccc}
s_{12} & s_{13} & s_{14} & s_{15} & s_{16} & s_{23} & s_{24} & \\
0 & 0 & 0 & 0 & 0 & 0 & 1 * & \\
& & & \vdots & & & & \\
& & & & & & & \\
s_{25} & s_{26} & s_{34} & s_{35} & s_{36} & s_{45} & s_{46} & s_{56} \\
1 & 1 & 0 & 0 & 0 & 1 & 1 & 1 \\
& & & \vdots & & & &
\end{array}\right]
\end{gathered}
$$

In addition to the logical diagnostic matrix, the faulty subnetworks could be also located by $F D_{f}(f=1,2,3)$ referred to as the logical diagnostic function.

$$
F D_{\mathrm{f}}=\left(\bigcap_{i=1}^{k} T_{f}^{i}\right) \cap\left(\bigcap_{i=1}^{k} \hat{T}_{f}^{i}\right)
$$

where $T_{f}^{i}$ or $\hat{T}_{f}^{i}(f=1,2,3 ; i=1,2,3 \ldots k)$ is refereed to as the logical tearing function at $T$, tearing.

$$
\begin{aligned}
& T_{f}^{i}=s_{j 1} \cap s_{j 2} \cap \cdots \cap s_{j m} \\
& \hat{T}_{f}^{i}=\hat{s}_{j 1} \cup \hat{s}_{j 2} \cup \cdots \cup \hat{s}_{j m}
\end{aligned}
$$

where $s_{j l}, s_{j 2}, \ldots s_{j m}$ are labels of the sub-networks under $f=1$ or of pseudo sub-networks under $f=2,3$ judged as fault-free at $T_{i}$ tearing, and $\hat{s}_{j 1}, \hat{s}_{j 2} \cdots \hat{s}_{j m}$ otherwise.

It is easily proved that the following conclusions are correct. $\left(1^{0}\right)$ If and only if only one of these matrices $L D_{f}(f$ $=1,2,3)$ contains only one column in which all elements are 1 and the remaining columns otherwise. The $f$ corresponding to this matrix correctly represents the number of faulty subnetworks, and this column correctly represents the fault location. $\left(2^{0}\right)$ Functions $F D_{f}(f=1,2,3)$ can be simplified by Boolean Algebra. Therefore, if and only if only one of these simplified functions contains all components in which only one must be a complemented term $\hat{s}_{j x}$, then the number and the locations of faulty sub-networks should be respectively represented by the $f$ and the $\hat{s}_{j x}$ in the logical diagnostic function $F D_{f}$.

\section{Diagnosability for Some Typical Circuits}

In this section, we shall compare the diagnosability of the intersected-torn search approach compared with the unified decomposition approach ${ }^{[2,3]}$ with some typical circuits. It is emphasized that the latter requires all incident nodes to be accessible, and the former has no such requirement ${ }^{[4]}$.

1) A ladder circuit, such as a Chebyshev filter should be a good example. We examine a five-section Chebyshev filter circuit simply, as shown in Fig. 5 .

According to the matrices $D_{1}$ and $D_{2}$ which are formed by the unified decomposition approach in Reference [3], there are some identical column sets in it, such as $C_{1}=\left\{s_{21}, s_{12}\right\}, C_{2}$ $=\left\{s_{4}, s_{43}\right\}, C_{3}=\left\{s_{13}, s_{23}\right\}$ and $C_{4}=\left\{s_{34}, s_{35}\right\}$. Thus, the maximum number of diagnosable faulty sub-networks $f$ should be 1 . Then the number $f$ by the intersected-torn search approach for Fig. 5 is 2 . The verifying process is omitted.

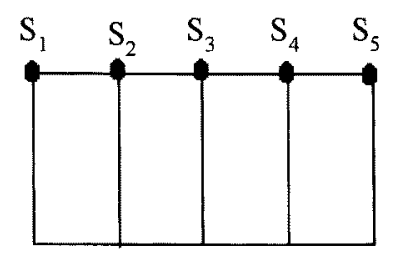

Fig. 5. Chebyshev filter TP 


$$
\begin{gathered}
D_{1}=\left[\begin{array}{lllll}
s_{1} & s_{2} & s_{3} & s_{4} & s_{5} \\
1 & 1 & 0 & 0 & 0 \\
0 & 1 & 1 & 0 & 0 \\
0 & 0 & 1 & 1 & 0 \\
0 & 0 & 0 & 1 & 1 \\
1 & 1 & 1 & 1 & 1
\end{array}\right] \\
D_{2}=\left[\begin{array}{lllllllllll}
s_{12} & s_{13} & s_{14} & s_{15} & s_{23} & s_{24} & s_{25} & s_{34} & s_{35} & s_{45} \\
1 & 1 & 1 & 1 & 1 & 1 & 1 & 0 & 0 & 0 \\
1 & 1 & 0 & 0 & 1 & 1 & 1 & 1 & 1 & 0 \\
0 & 1 & 1 & 0 & 1 & 1 & 0 & 1 & 1 & 1 \\
0 & 0 & 1 & 1 & 0 & 1 & 1 & 1 & 1 & 1 \\
1 & 1 & 1 & 1 & 1 & 1 & 1 & 1 & 1 & 1
\end{array}\right]
\end{gathered}
$$

2) Distribution feeder circuits. A diagram of a circuit is shown in Fig.6. According to the $D_{1}$ and $D_{2}$ matrices (to be omitted) by the unified decomposition approach, there are some identical column sets in it, such as $C_{1}=\left\{S_{2}, S_{12}\right\}, C_{2}$ $=\left\{\boldsymbol{S}_{3}, \boldsymbol{S}_{34}\right\}, C_{3}=\left\{\boldsymbol{S}_{6,}, \boldsymbol{S}_{56}\right\}$ and $C_{4}=\left\{\boldsymbol{S}_{7}, \boldsymbol{S}_{78}\right\}$. We can verify easily that the maximum number of faulty sub-networks diagnosed is 1 . But the number of tearing by the proposed approach is 6 under $f \leq 2$.

3) Cluster circuit. An example with two clusters is shown in Fig.7. It is easily verified that the maximum number of faulty sub-networks diagnosed by the unified decomposition approach is $f=1$, because there are some identical column sets in it, such as $C_{1}=\left\{\boldsymbol{s}_{2}, \boldsymbol{S}_{24}, \boldsymbol{S}_{25}\right\}, C_{2}=\left\{\boldsymbol{S}_{3}, \boldsymbol{S}_{36}, \boldsymbol{S}_{37}\right\}$. However, the number of tearing is never greater than six under $f \leq 2$ by the proposed approach. The verifying process is omitted.

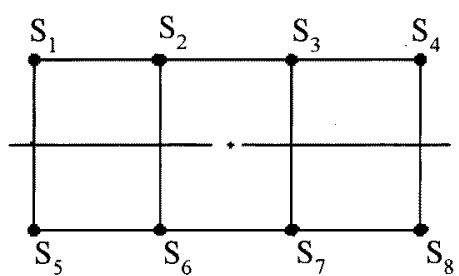

Fig. 6. Distribution feeder circuit TP

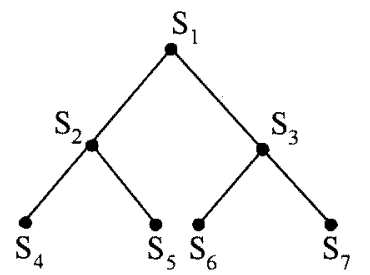

Fig. 7. Cluster circuit TP

\section{Example}

Fig. 8 shows a dc source electronic circuit. According to the construction of this circuit, it can be divided into five subnetworks. Its torn-diagnostic graph TP is identical with Fig.5. If the number of faulty sub-networks is two $(f \leq 2)$, then we have an intersected-torn search scheme satisfying the tearing rules as follows:

$$
\begin{array}{ll}
T_{1}: N_{1}^{1}=\left\{\mathrm{s}_{4}, \mathrm{~s}_{5}\right\}, & \hat{N}_{1}^{1}=\left\{\mathrm{s}_{1}, \mathrm{~s}_{2}, \mathrm{~s}_{3}\right\} ; \\
T_{2}: N_{1}^{2}=\left\{\mathrm{s}_{1}, \mathrm{~s}_{2}\right\}, & \hat{N}_{1}^{2}=\left\{\mathrm{s}_{3}, \mathrm{~s}_{4}, \mathrm{~s}_{5}\right\} ; \\
T_{3}: N_{1}^{3}=\left\{\mathrm{s}_{1}\right\}, & \hat{N}_{1}^{3}=\left\{\mathrm{s}_{2}, \mathrm{~s}_{3}, \mathrm{~s}_{4}, \mathrm{~s}_{5}\right\} ; \\
T_{4}: N_{1}^{4}=\left\{\mathrm{s}_{5}\right\}, & \hat{N}_{1}^{4}=\left\{\mathrm{s}_{1}, \mathrm{~s}_{2}, \mathrm{~s}_{3}, \mathrm{~s}_{4}\right\} .
\end{array}
$$

Let sub-networks $s_{2}$ and $s_{5}$ be faulty, the logical variables of these sub-networks set $N_{1}^{1}, \hat{N}_{1}^{1}, N_{1}^{2}, \hat{N}_{1}^{2}, N_{1}^{3}, \hat{N}_{1}^{3}, N_{1}^{4}$

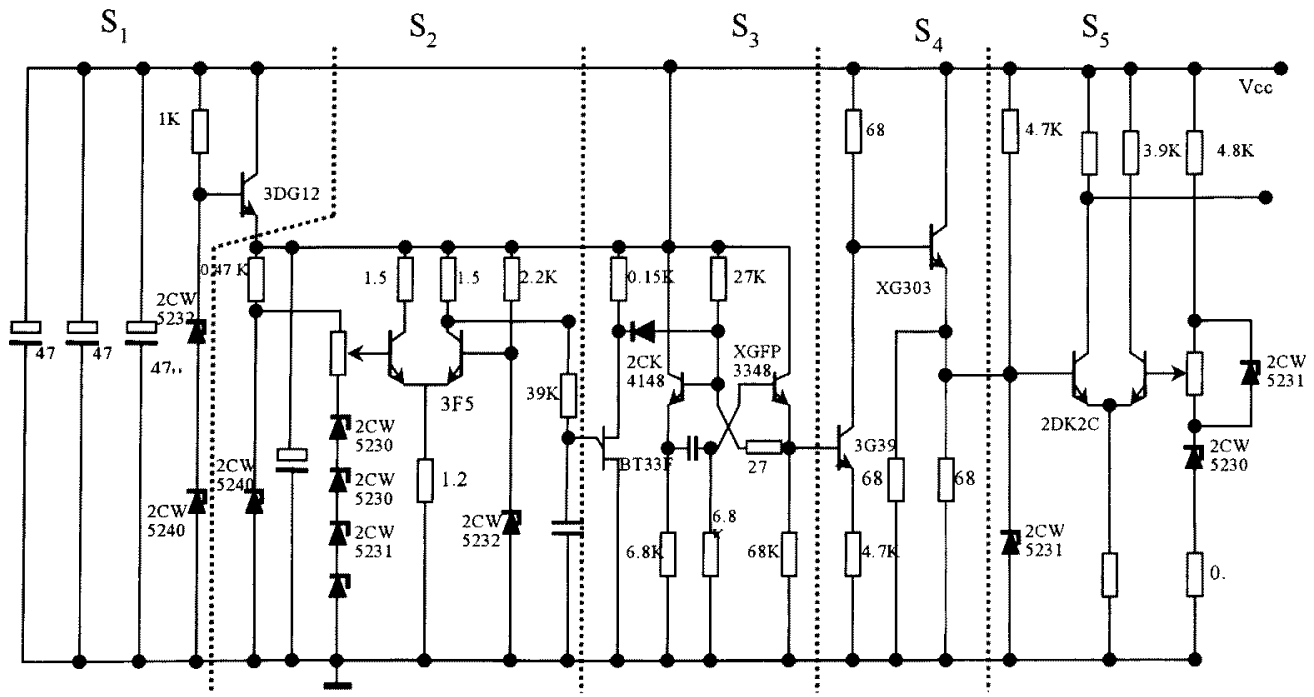

Fig. 8. Electronic circuit of a dc source 
and $\hat{N}_{1}^{4}$ should be following ${ }^{[5,7]}$ :

$$
\begin{array}{lll}
T_{1}: & \operatorname{TS}\left(N_{1}^{1}\right)=1, & \operatorname{TS}\left(\hat{N}_{1}^{1}\right)=1, \\
T_{2}: & \operatorname{TS}\left(N_{1}^{2}\right)=1, & \operatorname{TS}\left(\hat{N}_{1}^{2}\right)=1, \\
T_{3}: & \operatorname{TS}\left(N_{1}^{3}\right)=0, & \operatorname{TS}\left(\hat{N}_{1}^{3}\right)=1, \\
T_{4}: & \operatorname{TS}\left(N_{1}^{4}\right)=1, & \operatorname{TS}\left(\hat{N}_{1}^{4}\right)=1 .
\end{array}
$$

Based on the above logical variables, we can establish the logical diagnostic matrices $L D_{f}(f=1,2)$ as follows:

$$
L D_{1}=\left[\begin{array}{lllll}
s_{1} & s_{2} & s_{3} & s_{4} & s_{5} \\
1 & 1 & 1 & 1 & 1 \\
1 & 1 & 1 & 1 & 1 \\
0 & 1 & 1 & 1 & 1 \\
1 & 1 & 1 & 1 & 1
\end{array}\right]
$$$$
L D_{2}=\left[\begin{array}{cccccccccc}
s_{12} & s_{13} & s_{14} & s_{15} & s_{23} & s_{24} & s_{25} & s_{34} & s_{35} & s_{45} \\
0 & 0 & 1 & 1 & 0 & 1 & 1 & 1 & 1 & 0 \\
0 & 1 & 1 & 1 & 1 & 1 & 1 & 0 & 0 & 0 \\
0 & 0 & 0 & 0 & 1 & 1 & 1 & 1 & 1 & 1 \\
0 & 0 & 0 & 1 & 0 & 0 & 1 & 0 & 1 & 1
\end{array}\right]
$$

According to the above logical diagnostic matrices, the faulty sub-networks $S_{2}$ and $S_{5}$ can be identified. It is clear that this result is all just the same as the assumed condition. In addition to the logical diagnostic matrix, the faulty subnetworks could also be located by $F D_{f}(f=1,2)$ as follows:

$$
\begin{aligned}
F D_{1}= & S_{1} \cap\left(\bar{S}_{2} \cup \bar{S}_{3} \cup \bar{S}_{4} \cup \bar{S}_{5}\right) \cap\left(\bar{S}_{1} \cup \bar{S}_{2}\right. \\
& \left.\cup \bar{S}_{3} \cup \bar{S}_{4} \cup \bar{S}_{5}\right) \cap\left(\hat{S}_{1} \cup \hat{S}_{2} \cup \bar{S}_{3}\right. \\
& \left.\cup \bar{S}_{4} \cup \bar{S}_{5}\right) \cap\left(\bar{S}_{1} \cup \bar{S}_{2} \cup \bar{S}_{3} \cup \bar{S}_{4} \cup \bar{S}_{5}\right) \\
= & S_{1} \cap\left(\bar{S}_{2} \cup \bar{S}_{3} \cup \bar{S}_{4} \cup \bar{S}_{5}\right) \\
F D_{2}= & \left(S_{12} \cap S_{13} \cap S_{23} \cap S_{45}\right) \cap\left(S_{12} \cap S_{34}\right. \\
& \left.\cap S_{35} \cap S_{45}\right) \cap\left(S_{12} \cap S_{13} \cap S_{14}\right. \\
& \left.\cap S_{15}\right) \cap\left(S_{12} S_{13} \cap S_{14} \cap S_{23} \cap S_{24}\right. \\
& \left.\cap S_{34}\right) \cap\left(\bar{S}_{14} \cup \bar{S}_{15} \cup \bar{S}_{24} \cup \bar{S}_{25}\right. \\
& \left.\cup \bar{S}_{34} \cup \bar{S}_{35}\right) \cap\left(\bar{S}_{13} \cup \bar{S}_{14} \cup \bar{S}_{15}\right. \\
& \left.\cup \bar{S}_{23} \cup \bar{S}_{24} \cup \bar{S}_{25}\right) \cap\left(\bar{S}_{23} \cup \bar{S}_{24}\right. \\
& \left.\cup \bar{S}_{25} \cup \bar{S}_{34} \cup \bar{S}_{35} \cup \bar{S}_{45}\right) \cap\left(\bar{S}_{15}\right. \\
& \left.\cup \bar{S}_{25} \cup \bar{S}_{35} \cup \bar{S}_{45}\right) \\
= & S_{12} \cap S_{13} \cap S_{14} \cap S_{15} \cap S_{23} \cap S_{24} \cap \bar{S}_{25} \\
& \cap S_{34} \cap S_{35} \cap S_{45}
\end{aligned}
$$

According to the above logical diagnostic function $F D_{1}$ and $F D_{2}$, it is clear that the faulty sub-networks $S_{2}$ and $S_{5}$ can be identified.
This example shows that the faulty sub-networks can be identified by 4 tearings with the proposed approach. However, the unified decomposition approaches in Reference 2 and 3 cannot identify the faulty sub-networks due to equivocal diagnosis. It is also indicated that the proposed approach can diagnose large electronic circuits effectively.

\section{Conclusions}

In this paper, a new off-line intersected torn node search approach for fault diagnosis of analog electronic circuits at the sub-network level is proposed. This approach breaks through the limitation that all torn terminals must be accessible, and applies the computing-self-testing ways of the logical diagnostic function or logical diagnostic matrixes to judge whether the sub-network is faulty. An advantage is that the proposed approach reduces the range of fault diagnosis of large electronic circuits and the computing time of fault diagnosis. Another advantage is that the approach raises the re-applying efficiency of accessible nodes. Therefore, the proposed approach is fast and efficacious. In particular, some typical circuits, such as ladder circuits, distribution feeder circuits and cluster circuits and so on, are easily verified that the maximum number of faulty sub-networks diagnosed by the unified decomposition approach should be $f=1$. However, the maximum number by the proposed approach can be $f=2$. Thus, this application is more extensive than the unified decomposition approach. Therefore, the proposed method better satisfies the engineering needs for sub-network level

(Manuscript received Mar. 23, 2001, revised Jul. 1,2002)

\section{References}

(1) Zhihong You, et al.: "Analog System-Level Fault Diagnosis Based on a Symbolic Method in the Frequency Domain", IEEE Trans. on Instrumentation and Measurement, Vol.44, No.1, pp.28-35 (1995-1)

(2) Salama A E, et al: "A Unified Decomposition Approach for Fault Location in Large Analog Networks", IEEE Trans, on CAS, Vol.31, No.7, pp.609-621 (1984-7)

(3) T. Ozawa, et al.: "Diagnosability in the Decomposition Approach for Fault Location in Large analog Networks", IEEE Trans. on CAS, Vol.32, No 4, pp.415 416 (1985-4)

(4) H. Dongquan, et al: "Topological Conditions for Fault Diagnosability at sub-network-level", Proc. ISCAS'90, New Orleans, pp2240-2243 (1990)

(5) C. jingding et al.: "Interval Analysis of Adequate Selection of Accessible Nodes in a Tolerance Network", J. of Electronics, China, Vol. 17, No.4, pp.359-364 (1995)

(6) C. Wu, el al.: "Analog Fault Diagnosis with Failure Bounds", IEEE T. on Circuits and system, Vol 29, No.5, pp. 277-284 (1982-5)

(7) C. Jinding, and H. Dongquan: "Application of Interval Analysis for Fault Diagnosis in a Tolerance Sub-network level", Journal of Fuzhou University, China, Vol 22, No.3, pp.45-49 (1994-3) 
Cai Jinding (Non-member) was born in Quanzhou, Fujian,

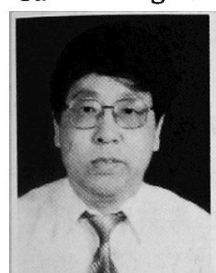

China, in 1954. He received his D. Eng. degree from $\mathrm{Xi}^{\prime}$ an Jiaotong University, China, in 2001. He is a professor at Fuzhou University, China. His research interests include large networks analysis and fault diagnosis, power system control and fault analysis. He is a member of Chinese Society

for Electrical Engineering

Chen Shuo (Member) was born in Fuzhou, Fujian, China, in

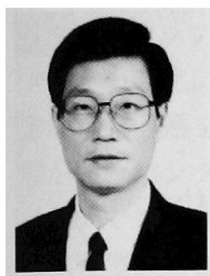

1964. He received the D. Eng. degree from Nagasaki University in 2000. $\mathrm{He}$ is an associate professor at Fuzhou University, China. His research interests are ac drives, control systems and mechatronics. He is a member of IEEE, the Japan Society of AEM, and Chinese Society for Electrical Engineering

Mineo Tsuji (Member) was born in Nagasaki, in 1953. He

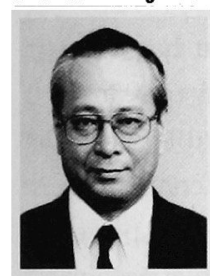
received the M.E. and Ph.D. degrees from Kyushu University in 1978 and 1981, respectively, both in electrical engineering. He became a lecture of Nagasaki University in 1981 and became a professor in 2001. His research interest is application of control theory in electrical system.
Ma Xikui (Non-member) was born in Xi' an, China, in 1958. He

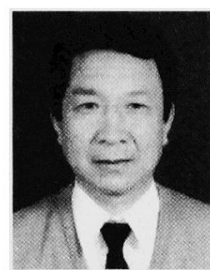
received the $\mathrm{M}$. S. degree from Xi' an Jiaotong University, China, in 1985. He is a professor of $\mathrm{Xi}$ an Jiaotong University. His research interests include networks theory and application of engineering electro- magnetic fields. $\mathrm{He}$ is a member of Chinese Society for Electrical Engineering

Huang Dongquan (Non-member) was born in Fuzhou, Fujian,

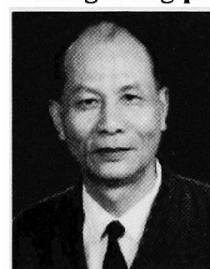
China, in 1930. He received his B. S. degree from Xi' an Jiaotong University in 1961 . He is a professor at Fuzhou University, China. His research interest includes the application of networks theory. He is a member of Chinese Society for Electrical Engineering 